\title{
BMJ Open Comparative effectiveness of first-line biological monotherapy use in rheumatoid arthritis: a retrospective analysis of the RECord-linkage On Rheumatic Diseases study on health care administrative databases
}

\author{
Ettore Silvagni, ${ }^{1}$ Alessandra Bortoluzzi, ${ }^{1}$ Greta Carrara, ${ }^{2}$ Anna Zanetti, ${ }^{2}$ \\ Marcello Govoni, ${ }^{1}$ Carlo Alberto Scirè ${ }^{1,2}$
}

To cite: Silvagni E, Bortoluzzi A, Carrara G, et al. Comparative effectiveness of first-line biological monotherapy use in rheumatoid arthritis: a retrospective analysis of the RECord-linkage On Rheumatic Diseases study on health care administrative databases. BMJ Open 2018;8:e21447. doi:10.1136/ bmjopen-2017-021447

- Prepublication history and additional material for this paper are available online. To view these files, please visit the journal online (http://dx.doi. org/10.1136/bmjopen-2017021447).

Received 29 December 2017

Revised 15 May 2018

Accepted 10 August 2018

Check for updates

(C) Author(s) (or their employer(s)) 2018. Re-use permitted under CC BY-NC. No commercial re-use. See rights and permissions. Published by BMJ.

${ }^{1}$ Department of Medical Sciences, Rheumatology Unit, University of Ferrara, Cona (Ferrara), Italy

${ }^{2}$ Epidemiology Unit, Italian Society for Rheumatology (SIR), Milan, Italy

Correspondence to

Dr Carlo Alberto Scirè;

c.scire@reumatologia.it

\section{ABSTRACT}

Objective These analyses aim to comparatively evaluate the persistence on treatment of different biological disease-modifying antirheumatic drugs (bDMARDs) when administered in monotherapy compared with combination with conventional synthetic disease-modifying antirheumatic drugs (csDMARDs) in rheumatoid arthritis (RA) patients receiving first-line biologics.

Design This is a retrospective observational study on Administrative Healthcare Databases.

Methods Data were extracted from healthcare databases of the Lombardy Region, Italy (2004-2013), as a part of the RECord-linkage On Rheumatic Diseases study, on behalf of the Italian Society for Rheumatology. Analyses included patients with RA starting first-line approved course of bDMARDs and evaluated drug survival by using Cox proportional hazard models. Results are presented as $\mathrm{HRs}$ and $95 \% \mathrm{Cl}$, crude and adjusted for prespecified confounders (age, sex, disease duration, Charlson Comorbidity Index (CCI), previous infections, use of concomitant glucocorticoids or non-steroidal antiinflammatory drugs (NSAIDs)).

Results 4478 patients with RA were included (17.84\% monotherapy). Etanercept, adalimumab and infliximab were the most prescribed first-line biologics. bDMARD monotherapy was associated with longer disease duration, higher $\mathrm{CCl}$, lower glucocorticoids and NSAIDs use. Compared with monotherapy, combination associated with a lower risk of failure (adjusted HR $0.79,95 \% \mathrm{Cl} 0.72$ to 0.88 ). Among monotherapies, considering etanercept as reference, adalimumab $(1.28,95 \% \mathrm{Cl} 1.03$ to 1.59$)$ and infliximab $(2.41,95 \% \mathrm{Cl} 1.85$ to 3.15$)$ had higher risk of failure. Concomitant methotrexate $(0.78,95 \% \mathrm{Cl}$ 0.70 to 0.87$)$, leflunomide $(0.80,95 \% \mathrm{Cl} 0.65$ to 0.98$)$ or csDMARD combinations $(0.77,95 \% \mathrm{Cl} 0.68$ to 0.87$)$ reduced the risk of bDMARD withdrawal.

Conclusion Adalimumab and infliximab monotherapies show lower retention rate compared with etanercept. The relatively small number of therapeutic courses different from tumour necrosis factor (TNF) inhibitors makes more difficult to achieve conclusive results with other
Strengths and limitations of this study

- This study provides results from administrative databases, following a previous study with the complete validation of classification algorithms for the identification of patients with rheumatoid arthritis (RA) at the population level through healthcare administrative databases.

- This study, as expected by study design, has no loss to follow-up and allows the analysis of a large sample of patients.

- Limitations of the RECord-linkage On Rheumatic Diseases study include the absence of specific disease clinical outcomes, in particular no information are available about disease activity and radiographic progression

biologics. Concomitant methotrexate, leflunomide and csDMARDs combination associate with longer survival on bDMARD. Our data confirm the effectiveness of the current practices in the choice of etanercept as first-line anti-TNF monotherapy and strengthen the currently recommended use of bDMARDs in combination with csDMARDs.

\section{INTRODUCTION}

Biological disease-modifying antirheumatic drugs (bDMARDs) are recommended in association with non-biological conventional synthetic disease-modifying antirheumatic drugs (csDMARDs) in the treatment of rheumatoid arthritis (RA). As stated by recent updated 2016 European League Against Rheumatism (EULAR) recommendations for the management of RA, ${ }^{1}$ bDMARDs should be combined with a csDMARD because of a superior efficacy of combination therapy. Among bDMARDs, recommendations suggest using tocilizumab (TCZ) when combination 
is not possible. Not only methotrexate (MTX) is useful in combination therapy but other csDMARDs can be also considered.

The better performance of bDMARD combination therapy with csDMARDs over bDMARD monotherapy has been clearly established both in terms of efficacy and retention rate. A recent meta-analysis of the Hazard Ratios (HRs) of bDMARDs discontinuation shows a 23\% lower risk of drug withdrawal for any cause in patients treated also with csDMARDs. ${ }^{2}$ A possible pharmacodynamic explanation is linked to an additive effect in the inhibitory profile of the combined drugs. In particular, differences between biologics exist and MTX plus adalimumab (ADA) inhibits more biological pathways compared with MTX plus TCZ, suggesting a synergistic effect of MTX in immunosuppression which differs across drugs. ${ }^{3}$ A pharmacokinetic effect of incremental doses of MTX in enhancing serum concentrations of ADA was also observed. ${ }^{4}$ Moreover, the immunogenicity of biologics, in terms of occurrence of antidrug antibodies, is lower in combination therapy and MTX reduces the incidence of the appearance of such antibodies. ${ }^{56}$ The effect of csDMARDs other than MTX in reducing immunogenicity of tumour necrosis factor TNF inhibitors (TNFis) is still unknown. TCZ and etanercept (ETA) share low immunogenicity ${ }^{78}$; MTX association did not influence the production of anti-TCZ ${ }^{9}$ and anti-abatacept (ABA) autoantibodies and, when autoantibodies occur, they are not associated with adverse events or discontinuation of therapy. ${ }^{10}$ Reduction in disability and radiographic progression are also superior in combination regimens. ${ }^{11}$

Limited data are available about the best biological treatment choice in real life when a biologic monotherapy is necessary for biological naive patients. In clinical practice, contraindications to MTX or early intolerance to csDMARDs are frequently observed, and clinicians need to start a biological monotherapy in these cases; the result is that patients with RA are treated with monotherapy nearby in one-third or even more cases. ${ }^{12-16}$

Differently from randomised controlled trials (RCTs), data from observational studies or registries explore the utilisation of monotherapy in real-life clinical practice ${ }^{2}$ and persistence in therapy is considered a good indirect and composite measure of effectiveness, safety and tolerability, reflecting the long-term impact on the course of the disease. Data of real-life overall persistence show that monoclonal TNFis are burdened by a higher risk of drug failure compared with ETA. ${ }^{2}$ Limited data are available for non-TNFis.

Objective of this analysis was to assess, in patients with RA receiving first-line approved biological therapy, the comparative effectiveness (expressed in terms of drug survival) of different bDMARDs when administered in monotherapy compared with combination therapy, accordingly to real-life clinical practice and in compliance with local regulatory approvals. Secondary objectives were to characterise features of patients starting monotherapy and to evaluate the specific effect of MTX combination therapy compared with other csDMARDs association regimens in determining persistence of bDMARD cotherapy.

To answer these questions, we took advantage by the RECord-linkage On Rheumatic Diseases (RECORD) dataset, including data from administrative health database (AHD) of the Lombardy region (Italy), analysing bDMARDs and concurrent drug exposures of all the first courses of bDMARDs of patients with RA between 2004 and 2013.

\section{MATERIALS AND METHODS \\ Study design and setting}

This is a retrospective observational study on AHD of Lombardy Region, Italy (>10000 000 inhabitants). Access to data was granted by the General Directorate of Health for the purpose of the RECORD study, a project promoted by the Italian Society for Rheumatology aiming to set up a national surveillance system to monitor the health burden of rheumatic diseases in Italy using AHD. The protocol was approved by the ethical committee of the Pavia University Hospital. Data included were retrieved between 1 January 2004 and 31 December 2013.

\section{Patient and public involvement}

This is a retrospective study based on AHD; patients were not directly involved in the research.

\section{Participants and variables}

The design of the RECORD study includes a database population of patients with RA and four age-matched and sex-matched controls from the general population. Patients with RA were identified through copayment exemption code 006.714.0, based on its previously demonstrated high specificity $(96.39 \%)$ and high sensitivity $(77.08 \%)$ for RA, ${ }^{17}$ in line with other studies following a similar methodology. ${ }^{18} 19$

Study population was defined among patients with RA and at least one delivery of first-line approved bDMARDs (ABA, ADA, certolizumab (CTZ), ETA, golimumab (GOL), infliximab (INF) and TCZ). Rituximab (RTX) was excluded due to the local limitation in first-line deliverability of this drug in patients with RA. The exposure to non-steroidal anti-inflammatory drugs (NSAIDs), daily mean glucocorticoid (GC) dosage (expressed in terms of prednisone equivalent, milligrams per day) and to specific csDMARDs (MTX, leflunomide (LFN), cyclosporin A (CYA), hydroxychloroquine (HCQ) or sulfasalazine (SSZ) ) was defined by the drug delivery recorded in the administrative database.

Data included demographics (birth date, gender, death date or embarkment, drug delivery (Anatomic Therapeutic Chemical) code, date of drug delivery, quantity), exemptions (exemption code, date of exemption), outpatient services (code and date) and hospital discharge forms including information on beginning and end of hospitalisation, International Classification of Disease, Ninth revision, Clinical Modification diagnoses and 
Disease Related Group. Previous bacterial infections were considered if hospitalisation for bacterial infection or an antibiotic treatment course of over 14 days occurred in the previous year. ${ }^{20}$

\section{Statistical methods}

The primary outcome was persistence with first-line bDMARD, which was defined as the length of time between drug delivery plus drug coverage. A patient was considered exposed to a specific treatment from the first prescription of drug until the last one plus 6 months, in order to consider the coverage period of drug also after its withdrawal, or until the first prescription of the subsequent drug. Censoring was defined at treatment stop date plus drug coverage or until the start of a new bDMARD, death or at the end of established follow-up, whichever came first. Drug persistence in bDMARD therapy was compared using Cox proportional hazard models. Results were presented as HR and $95 \% \mathrm{CI}$, crude and adjusted for prespecified confounders (sex, age, disease duration, Charlson Comorbidity Index (CCI) ${ }^{21}$ concomitant use of NSAIDs, GCs average dosage and previous bacterial infections). A secondary analysis, focused on the role of each associated csDMARD in bDMARD persistence, was analysed by the same mechanism (first considering combination biologics as a whole and then investigating the interaction between different csDMARDs and each bDMARD). A sensitivity analysis was performed to investigate if different periods of bDMARDs prescription could have influenced persistence data (a distinction was made before and after 31 December 2009, according to changes occurred in local bDMARDs deliverability).

All the analyses were performed using the Stata11 software (STATA Corporation, College Station, Texas, USA) and R statistical Software (Foundation for Statistical Computing, Vienna, Austria).

\section{RESULTS}

\section{Study population and descriptive data}

A total of 4478 patients with RA who had their first-line bDMARD delivery were included (13728 person/time), of which 3472 were women (77.53\%); mean age (SD) at bDMARD exposure was 55.48 (12.69) years with a modal disease duration of over 5 years. No missing data nor lostto-follow-up were recorded, nor expected by design. A mean (SD) CCI of $1.16(0.48)$ was observed (table 1 ).

bDMARD monotherapy was administered to 799 patients $(17.84 \%)$, while $3679(82.16 \%)$ experienced csDMARDs association. Most prescribed bDMARDs were ETA (1787 patients, 39.91\%), ADA (1143, 25.52\%) and INF $(861,19.23 \%)$. ETA was the most prescribed drug out of monotherapy (385 patients, $48.19 \%$ ) and in combination group (1402 patients, 38.11\%) (table 2).

Among concomitant csDMARD therapy, MTX was the most commonly prescribed (2297 patients had only concurrent MTX, 62.44\%; 223 only concurrent LFN (6.06\%), 151 concurrent HCQ (4.10\%), 43 SSZ (1.17\%),
41 CYA $(1.11 \%)) .924$ patients $(25.12 \%)$ experienced a combination of different csDMARDs; in this group, a total of 827 patients received MTX, 254 LFN, 131 SSZ, 619 HCQ and 116 CYA.

451 bDMARD monotherapies were started before 31 December 2009 (252 ETA, 136 ADA, 62 INF, 1 ABA, no TCZ, CTZ and GOL) and 348 after 1 January 2010 (133 ETA, 65 ADA, 47 TCZ, 33 ABA, 30 CTZ, 21 GOL and 19 INF).

\section{Factors influencing monotherapy}

Monotherapy was associated with longer disease duration, a higher CCI (in particular hepatic and renal disease and heart failure), lower use of GCs and NSAIDs (table 1).

\section{Risk of bDMARD failure}

Compared with monotherapy, combination with at least one csDMARD was associated with a lower risk of drug failure (crude HR 0.77 , 95\% CI 0.69 to 0.85 ; adjusted HR $0.79,95 \%$ CI 0.72 to 0.88). Among patients in bDMARD monotherapy, considering ETA as reference, the adjusted HR for bDMARD failure was 1.28 for ADA (95\% CI 1.03 to 1.59 ) and 2.41 for INF (95\% CI 1.85 to 3.15) (figure 1); ABA monotherapy was associated with a reduced-but not statistically significant—risk of failure, while TCZ was almost equal to ETA. Otherwise, among combination therapies, only INF was significantly inferior compared with ETA monotherapy. The risk of failure evaluated for the other bDMARDs was not statistically different from ETA monotherapy.

\section{Influence of different csDMARDs in persistence in bDMARD treatment}

Considering specific combination therapy and taking bDMARD monotherapy as reference, concurrent csDMARDs significantly reduced the risk of bDMARD withdrawal (adjusted HR 0.78 for MTX alone, 95\% CI 0.70 to 0.87 ; HR 0.80 for LFN alone, $95 \%$ CI 0.65 to 0.98 ; HR 0.77 for combination of different csDMARDs, $95 \%$ CI 0.68 to 0.87 ) (figure 2), while no statistical significant improvement in drug survival was observed for SSZ, HCQ or CYA when used as the single associated csDMARD.

The analysis of different csDMARDs in determining persistence of different bDMARD treatment showed that MTX alone or in combination with other csDMARDs positively influenced persistence in INF treatment, while other associations between csDMARDs and bDMARDs did not significantly modify the concomitant biological drug survival.

\section{Sensitivity analysis}

After stratification in different periods of time (before and after 31 December 2009), an increase in the previous reported risk of drug failure for INF was observed (HR 2.72, 95\% CI 1.51 to 4.90). The risk of ADA failure remains elevated compared with ETA monotherapy, but differences according to the time period were no longer significant (HR 1.17, 95\% CI 0.70 to 1.96); the risk of failure for other bDMARDs remains not significantly different from 
Table 1 Clinical and demographic features of the study population including 4478 patients with RA and their distribution in bDMARDs monotherapy and combination therapy

Study population Monotherapy ( $\mathrm{N}=799)$ Combination ( $\mathrm{N}=3679)$ P values

\begin{tabular}{|c|c|c|c|c|}
\hline \multicolumn{5}{|l|}{ Demographic characteristics } \\
\hline Mean age (SD, years) & $55.48(12.69)$ & $54.90(12.97)$ & $55.61(12.62)$ & 0.136 \\
\hline Female, n (\%) & $3472(77.53)$ & $614(76.85)$ & $2858(77.68)$ & 0.607 \\
\hline \multicolumn{5}{|l|}{ Clinical characteristics } \\
\hline \multicolumn{5}{|l|}{ Disease duration, $\mathrm{n}(\%)$} \\
\hline$<1$ years & $1028(22.96)$ & $153(19.15)$ & $875(23.78)$ & \multirow[t]{4}{*}{$<0.001$} \\
\hline$>1$ to $\leq 2$ years & $1106(24.7)$ & $188(23.53)$ & $918(24.95)$ & \\
\hline$\geq 3$ to $\leq 5$ years & $1064(23.76)$ & $171(21.40)$ & $893(24.27)$ & \\
\hline$>5$ years & $1280(28.58)$ & $287(35.92)$ & $993(26.99)$ & \\
\hline Number of comorbidities=0, $n(\%)$ & $3941(88.01)$ & $683(85.48)$ & $3258(88.56)$ & \multirow[t]{4}{*}{0.004} \\
\hline Number of comorbidities=1, $n(\%)$ & $416(9.29)$ & $80(10.01)$ & $336(9.13)$ & \\
\hline Number of comorbidities=2, $n(\%)$ & $105(2.34)$ & $30(3.75)$ & $75(2.04)$ & \\
\hline Number of comorbidities $\geq 3, n(\%)$ & $16(0.36)$ & $6(0.75)$ & $10(0.27)$ & \\
\hline Charlson Comorbidity Index ${ }^{\star}$, mean (SD) & $1.16(0.48)$ & $1.22(0.60)$ & $1.15(0.45)$ & 0.009 \\
\hline CHD, n (\%) & $66(1.47)$ & $16(2.00)$ & $50(1.36)$ & 0.193 \\
\hline Heart failure, n (\%) & $12(0.27)$ & $5(0.63)$ & $7(0.19)$ & 0.047 \\
\hline Vascular pathology, n (\%) & $10(0.22)$ & $4(0.50)$ & $6(0.16)$ & 0.086 \\
\hline Dementia, n (\%) & $1(0.02)$ & $1(0.13)$ & $0(0.00)$ & 0.178 \\
\hline COPD, n (\%) & 49 (1.09) & $11(1.38)$ & $38(1.03)$ & 0.451 \\
\hline Mild hepatic disease †, N (\%) & $73(1.63)$ & $24(3.00)$ & $49(1.33)$ & 0.002 \\
\hline Diabetes, n (\%) & $276(6.16)$ & $41(5.13)$ & $235(6.39)$ & 0.195 \\
\hline Renal disease, $\mathrm{n}(\%)$ & $32(0.71)$ & $18(2.25)$ & $14(0.38)$ & $<0.001$ \\
\hline Neoplasm ${ }^{\dagger}, \mathrm{n}(\%)$ & $67(1.50)$ & $16(2.00)$ & $51(1.39)$ & 0.198 \\
\hline Leukaemia/lymphoma, n (\%) & $1(0.02)$ & $1(0.13)$ & $0(0.00)$ & 0.178 \\
\hline Previous infections, $\mathrm{n}(\%)$ & $822(18.36)$ & $140(17.52)$ & $682(18.54)$ & 0.501 \\
\hline Concomitant NSAIDs, n (\%) & $3386(75.61)$ & $485(60.70)$ & $2901(78.85)$ & $<0.001$ \\
\hline Concomitant GCs, n (\%) & $3045(68.00)$ & $428(53.57)$ & $2617(71.13)$ & $<0.001$ \\
\hline GCs dose (mg/day), mean (SD) & $2.23(3.08)$ & $1.85(3.32)$ & $2.31(3.01)$ & $<0.001$ \\
\hline
\end{tabular}

*Diabetes with end-organ damage, AIDS, cerebrovascular disease, peptic ulcer and hemiplegia are not shown due to absence of cases in monotherapy group.

†Severe hepatic disease and metastatic neoplasms are not shown due to absence of cases in both groups.

bDMARD, biological disease-modifying antirheumatic drug; CHD, coronary heart disease; COPD, chronic obstructive pulmonary disease; GC, glucocorticoid; NSAIDs, non-steroidal anti-inflammatory drugs; RA, rheumatoid arthritis.

ETA (online supplementary file 1). A subanalysis of the patients receiving ADA monotherapy after 1 January 2010 showed that in this group a proportional higher number of males was present $(\mathrm{p}=0.004)$, with longer disease duration $(\mathrm{p}<0.001)$ and higher CCI $(\mathrm{p}=0.018)$.

\section{DISCUSSION}

Using bDMARDs as monotherapy in clinical settings is a common practice for patients with RA and recognised by health authorities although current guidelines recommend combining them with csDMARDs. The aims of this study were to describe persistence and factors associated with starting biological monotherapy in a realworld setting. In our study, monotherapy was common and observed in approximately one out of five biological naive patients with RA initiating a biological agent $(17.8 \%)$. In previous biologics registries and claims database studies, $12 \%-39 \%$ of patients were taking biologics as monotherapy. ${ }^{22-24}$

From a practical perspective, it seems even more important to investigate those factors which may drive prescribing monotherapy. Indeed, bDMARDs monotherapy could be representative of a subgroup of patients with a more difficult disease management. ${ }^{25}$ It has been reported that older patients, with longer disease duration and multiple comorbidities, lower body mass index and higher disease activity show higher probability to undergo monotherapy. ${ }^{16222526}$ Concomitant use of GCs 
Table 2 Distribution of different bDMARDs in monotherapy and combination therapy with csDMARDs

\begin{tabular}{lccc}
\hline bDMARDS & $\begin{array}{l}\text { Study } \\
\text { population }\end{array}$ & $\begin{array}{l}\text { Monotherapy } \\
\text { (N=799) }\end{array}$ & $\begin{array}{l}\text { Combination } \\
\text { (N=3679) }\end{array}$ \\
\hline ABA, n (\%) & $189(4.22)$ & $34(4.26)$ & $155(4.21)$ \\
\hline ADA, n (\%) & $1143(25.52)$ & $201(25.16)$ & $942(25.60)$ \\
CTZ, n (\%) & $156(3.48)$ & $30(3.75)$ & $126(3.42)$ \\
ETA, n (\%) & $1787(39.91)$ & $385(48.19)$ & $1402(38.11)$ \\
GOL, n (\%) & $151(3.37)$ & $21(2.63)$ & $130(3.53)$ \\
\hline INF, n (\%) & $861(19.23)$ & $81(10.14)$ & $780(21.20)$ \\
\hline TCZ, n (\%) & $191(4.27)$ & $47(5.88)$ & $144(3.91)$ \\
\hline
\end{tabular}

ABA, abatacept; ADA, adalimumab; bDMARD, biological disease-modifying antirheumatic drug; csDMARD, conventional synthetic disease-modifying antirheumatic drug; CTZ, certolizumab; ETA, etanercept; GOL, golimumab; INF, infliximab; TCZ, tocilizumab.

predicts higher bDMARD discontinuation, reflecting a much severe course of the disease. ${ }^{223}$ Accordingly, in our retrospective study based on AHD, we have observed a significant association between monotherapy and longer disease duration and a higher number of comorbidities. As expected, hepatic and renal diseases were the most limiting factors for csDMARDs association. NSAIDs and GCs were negatively associated with monotherapy, likely reflecting contraindication to these drugs due to concomitant comorbidities.

As demonstrated by the majority of published reallife studies and RCTs, our study confirms that bDMARD risk of failure is significantly lower in combination with csDMARDs (21\% lower risk of drug withdrawal compared with monotherapy). Concerning monotherapy, in a Swiss study of retention rate which analyses data from Swiss Clinical Quality Management Registry between 2004 and $2013,{ }^{25} 27 \%$ of all biologics therapeutic courses was initiated as monotherapy (the higher percentage of monotherapy was for CTZ with 46\%; 35\% ETA; 35\% TCZ; $29 \%$ ABA; 26\% ADA; 23\% RTX; 17\% GOL and 14\% INF) and a further $13 \%$ experienced a transient phase of monotherapy overtime; discontinuation of bDMARD occurred in $63 \%(1545 / 2453)$ and the adjusted HR for discontinuation of biological monotherapy versus combination was 1.15 (95\% CI 1.03 to $1.30, \mathrm{p}=0.018)$, although differences between the two groups were relatively modest.

\begin{tabular}{|c|c|}
\hline & Hazard Ratio $(9$ \\
\hline \multicolumn{2}{|l|}{ ETA + csDMARD } \\
\hline Crude & $0.78(0.67-0.90)$ \\
\hline Adjusted & $0.76(0.66-0.89)$ \\
\hline \multicolumn{2}{|l|}{ ADA monotherapy } \\
\hline Crude & $1.45(1.17-1.79)$ \\
\hline Adjusted & $1.28(1.03-1.59)$ \\
\hline \multicolumn{2}{|l|}{$A D A+C S D M A R D$} \\
\hline Crude & $0.97(0.83-1.13)$ \\
\hline Adjusted & $0.99(0.85-1.15)$ \\
\hline \multicolumn{2}{|l|}{ INF monotherapy } \\
\hline Crude & $2.83(2.17-3.69)$ \\
\hline Adjusted & $2.41(1.85-3.15)$ \\
\hline \multicolumn{2}{|l|}{ INF + cSDMARD } \\
\hline Crude & $1.22(1.05-1.43)$ \\
\hline Adjusted & $1.21(1.03-1.41)$ \\
\hline \multicolumn{2}{|l|}{ GOL monotherapy } \\
\hline Crude & $2.01(0.95-4.28)$ \\
\hline Adjusted & $1.87(0.88-3.98)$ \\
\hline \multicolumn{2}{|l|}{ GOL + CSDMARD } \\
\hline Crude & $1.32(0.98-1.78)$ \\
\hline Adjusted & $1.26(0.94-1.70)$ \\
\hline \multicolumn{2}{|l|}{ CTZ monotherapy } \\
\hline Crude & $1.41(0.69-2.85)$ \\
\hline Adjusted & $1.36(0.67-2.75)$ \\
\hline \multicolumn{2}{|l|}{ CTZ + CSDMARD } \\
\hline Crude & $1.01(0.71-1.43)$ \\
\hline Adjusted & $0.97(0.68-1.38)$ \\
\hline \multicolumn{2}{|l|}{ ABA monotherapy } \\
\hline Crude & $0.55(0.23-1.34)$ \\
\hline Adjusted & $0.53(0.22-1.28)$ \\
\hline \multicolumn{2}{|l|}{$A B A+C S D M A R D$} \\
\hline Crude & $0.81(0.58-1.13)$ \\
\hline Adjusted & $0.74(0.53-1.03)$ \\
\hline \multicolumn{2}{|l|}{ TCZ monotherapy } \\
\hline Crude & $1.06(0.62-1.81)$ \\
\hline Adjusted & $1.02(0.59-1.75)$ \\
\hline \multicolumn{2}{|l|}{$\mathrm{TCZ}+$ CSDMARD } \\
\hline Crude & $1.10(0.83-1.47)$ \\
\hline Adjusted & $1.13(0.85-1.50)$ \\
\hline
\end{tabular}

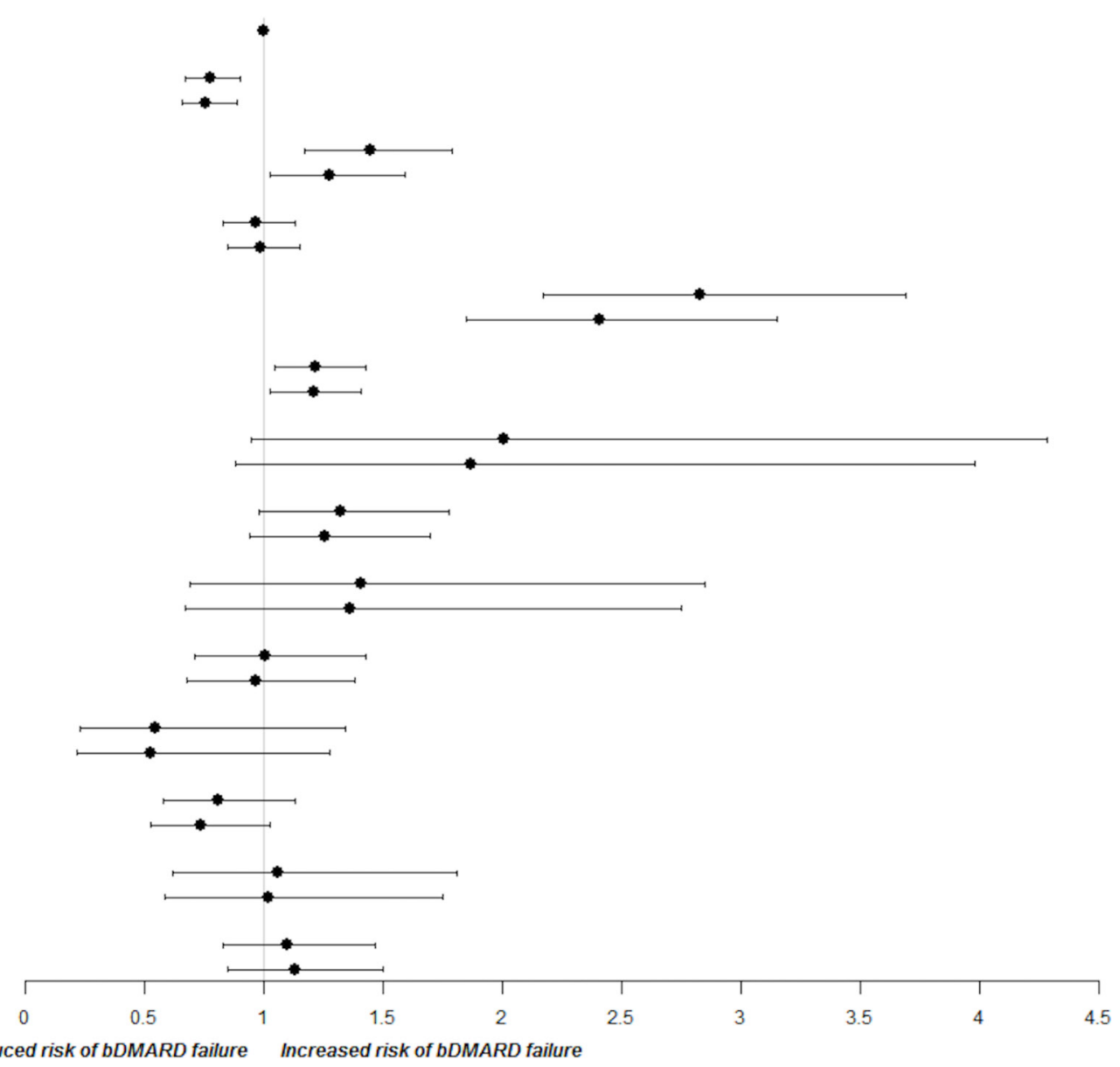

Figure 1 Crude and adjusted $\mathrm{HR}$ and $95 \% \mathrm{Cl}$ for bDMARD failure when administered in first-line monotherapy and in combination with csDMARDs. ABA, abatacept; ADA, adalimumab; bDMARD, biological disease-modifying antirheumatic drug; csDMARD, conventional synthetic disease-modifying antirheumatic drug; CTZ, certolizumab; ETA, etanercept; GOL, golimumab; INF, infliximab; TCZ, tocilizumab. 
bDMARD monotherapy

Combination with any csDMARD

Crude

Adjusted

Combination with MTX

Crude

Adjusted

Combination with LFN

Crude

Adjusted

Combination with SSZ

Crude

Adjusted

Combination with $\mathrm{HCQ}$

Crude

Adjusted

Combination with CYA

Crude

Adjusted

Combination with combination of $\geqslant 1$ csDMARD

Crude

$0.79(0.70-0.89)$
$0.77(0.68-0.87)$

Adjusted

$0.79(0.70-0.89)$
$0.77(0.68-0.87)$

Reference

$0.77(0.69-0.85)$

$0.79(0.72-0.88)$

$0.73(0.66-0.82)$

$0.78(0.70-0.87)$

$0.77(0.63-0.94)$

$0.80(0.65-0.98)$

$0.87(0.60-1.26)$

$0.92(0.63-1.34)$

$1.01(0.80-1.26)$

$0.98(0.78-1.23)$

$1.45(1-2.11)$

$1.36(0.93-1.98)$

Hazard Ratio $(95 \% \mathrm{Cl})$

Reduced risk of bDMARD failure Increased risk of bDMARD failure

Figure 2 Crude and adjusted HR and 95\% Cl for different csDMARDs in determining the risk of first-line bDMARD failure. bDMARD, biological disease-modifying antirheumatic drug; csDMARD, conventional synthetic disease-modifying antirheumatic drug; CYA, ciclosporin A, HCQ, hydroxychloroquine; LFN, le flunomide; MTX, methotrexate; SSZ, sulfasalazine.

Treatment failure was influenced not only by the type of BDMARD but even by gender, number of previous bDMARDs, year of initiation of the receiving drug, seropositivity, disease duration and activity. TNFis strongly impact these data, being historically the first bDMARD entered in clinical practice and accounting for about $80 \%$ of therapeutic courses; therefore, conclusive results are still lacking. Overall, the type of bDMARD is certainly one of the most important factors influencing persistence, and INF monotherapy is burdened by the higher rate of withdrawal. ${ }^{2} 2327$ Globally, monoclonal antibodies against TNF- $\alpha$ share higher discontinuation compared with $\mathrm{ETA}^{228}$; whether the global higher immunogenicity of monoclonal antibodies is strictly responsible for this difference is still matter of debate. In an observational study, Kristensen et $a l^{29}$ stand out a higher adherence in first-line ETA-treated patients compared with INF; concomitant MTX was associated with better persistence in both groups but significantly higher for ETA. In a 12-year retention rate study of first-line TNFi, Favalli $e t a l^{30}$ demonstrated a higher risk of drug failure for ADA (HR 2.89, $95 \%$ CI 2.2 to 3.78 ) and INF (HR 2.56, 95\% CI 1.92 to
3.4) compared with ETA, similarly to what is reported by a French multicentric study by Frazier-Mironer ${ }^{31}$ and the GISEA registry $^{32}$; MTX users in combination with biologics shared higher retention rate compared with TNFi monotherapy (HR 1.48, 95\% CI 1.18 to 1.86). Jorgensen et $^{2} l^{23}$ analysed 775 patients in the Danish registry and showed that persistence in monotherapy was significantly higher for all biologics compared with INF (HR of withdrawal 2.53 for INF compared with other bDMARDs, 95\% CI 1.70 to $3.77, \mathrm{p}<0.001$ ), and these features were independent of the number of previous biologics. In German RABBIT registry, a longer persistence was found in combination therapy with TNFi but remission rates were not significantly different from monotherapy group. ${ }^{14}$ The south Swedish SSATG registry ${ }^{33}$ evaluated differences in biologics monotherapy persistence in different biologics courses over 6 years and highlighted a significant difference among bDMARDs with highest retention rates observed for RTX and ETA. In RADIUS registry, ${ }^{34}$ which analysed different efficacy between ETA, INF and csDMARDs therapy, patients receiving either ETA plus MTX (adjusted OR 1.29, 95\% CI 1.09 to $1.52, \mathrm{p}<0.01$ ) or 
ETA monotherapy (OR 1.23, 95\% CI 1.02 to 1.47 , p<0.05) were more likely to achieve a modified ACR20 response at 12 months than patients receiving MTX alone, INF plus MTX or INF alone; persistence in therapy however was higher for INF plus MTX ( $71 \%$ of INF group persisted in therapy after 12 months versus $69 \%$ of ETA monotherapy, $67 \%$ INF alone and 61\% ETA plus MTX); noteworthy cost of the therapy was claimed as a significant cause of discontinuation of biological therapy in this registry (up to $6 \%$ of ETA monotherapy changed treatment due to high costs). The ACT-iON observational study ${ }^{35}$ explored different persistence rates among first-line TNFis and TCZ and showed a better persistence for TCZ compared with TNFis as a whole; a comparison between first-line monotherapies was not possible due to the low number of cases. Our data are in keeping with current literature and show a lower persistence for first-line monoclonal TNFis (ADA and INF) monotherapy compared with ETA monotherapy, suggesting that these bDMARDs should be avoided when a TNFi monotherapy is thought to be necessary.

Data from RTCs confirm this tendency among TNFis, either in terms of retention rate as well for radiological outcomes, but long-term head-to-head comparative trials among different bDMARDs specifically designed to test this outcome are lacking. With regard to non-TNFis, data about a real superiority of combination therapy compared with monotherapy are controversial. ${ }^{76} \mathrm{~A}$ pan-European analysis of registries including nearby 3400 patients showed that retention of ABA was not influenced by csDMARDs co-therapy. ${ }^{7}$ TCZ has gained the reputation to be the best bDMARD to use in monotherapy and the only one with a satisfactory durability ${ }^{121336}$ and cost-effectiveness. ${ }^{37}$ However, also for this drug, association strategy has demonstrated to be useful in clinical trials giving some advantages when compared with monotherapy. In ACT-RAY trial, after 2 years, a difference in radiographic progression was observed favouring combination regimen with $\mathrm{MTX}^{38}$ and, in a recent post hoc subanalysis of ACT-SURE study, concomitant csDMARDs helped to achieve low disease activity earlier than in TCZ monotherapy, ${ }^{39}$ similarly to what demonstrated by Kaneko et al in SURPRISE study. ${ }^{40}$ Conversely, other studies did not show particular advantages in terms of clinical efficacy of TCZ combination therapy over monotherapy. ${ }^{41} 42$ In ADACTA study, ${ }^{43}$ TCZ monotherapy reduced significantly disease activity score and Clinical Disease Activity Index compared with ADA monotherapy after 24 weeks, in a head-to-head comparison between monotherapies. Our study confirms a similar persistence rate between non-TNFi monotherapy compared with combination, but the small size of our sample and the calendar-period of the analysis does not allow a conclusive remark.

Our data show that either MTX or LFN or combination of different csDMARDs significantly increase bDMARDs persistence rate, while CYA is associated with a higher (but not significant) rate of drug failure. Previous reports on the benefits of combining different csDMARDs with
bDMARDs have shown contrasting results; Soliman $e t a l^{16}$ explored the role of different csDMARDs intervention in biological persistence in a real-life study which evaluated persistence in over 10000 patients from a British registry and stated that MTX combination was linked to a better persistence of the first TNFi when compared with no csDMARDs, LFN or SSZ, but the best overall persistence was seen among patients receiving TNFi in combination to MTX and either SSZ or HCQ or both, in line with our results. Similarly, Manders et al found a similar persistence rate in TNFi plus MTX group compared with TNFi plus MTX plus others. ${ }^{44}$ De Stefano et $a t^{45}$ reported a similar efficacy and safety profile for TNFi combined with either MTX or LFN in early RA, but univocal data for LFN combination to bDMARDs are lacking and limited by the high number of associations with INF. ${ }^{46}$ Conversely, Kristensen $e t a l^{29}$ demonstrated that concomitant MTX, but not other csDMARDs, was associated with a better persistence with first-line ETA or INF therapy, but significantly higher for ETA. A positive influence in terms of efficacy has been observed for LFN combined with RTX in GERINIS study ${ }^{47}$ and in CERERRA collaboration. ${ }^{48}$

When stratifying by calendar year-subclasses, after the introduction of other bDMARDs in current recommended therapeutic approach, the risk of failure for INF monotherapy slightly increased, while ADA monotherapy became not statistically different from ETA. Higher number of comorbidities and longer disease duration in this subgroup could reflect the selection of a particular subset of patients for whom an acceptance of a suboptimal control of disease activity has been made, despite a real efficacy of the drug. Afterwards, the reduction of the sample size after this stratification could have influenced the results as well as the prescription attitude of bDMARDs might have changed during the period of analysis. In fact, as shown by literature, ${ }^{225}$ the year of treatment could have influenced bDMARD retention rate, since rheumatologists are more prone to change biologics if more alternatives are available,${ }^{27}$ although data about this issue are controversial. ${ }^{49}$

Our study has some limitations. The different burden of prescribed bDMARDs (being ETA, ADA and INF the most prescribed ones) could have influenced our results; to this regard, GOL, CTZ and non-TNFis associated with a lower prescription rate and RTX was excluded due to the local limitation in first-line deliverability. This limitation makes conclusions not generalisable for all biological agents. We adjusted for prespecified confounders but confounding of unmeasured factors could not be excluded (50), for example, other comorbidities different from those included in CCI, previous csDMARDs treatment history, changed treatment behaviour overtime with different GCs and NSAIDs utilisation schemes or specific musculoskeletal disease or patients-related characteristics (radiological features, concomitant osteoarthritis, crystal arthropathies or fibromyalgia). Furthermore, the design of the study could not differentiate between patients starting monotherapy 'ab initio' and those reaching 
such monotherapy by a 'step-down' process; despite this behaviour accounts for only a small proportion, ${ }^{25}$ the overall prevalence could have been under-recognised due to specific design of trials and 'real-life' databases ${ }^{16}$; anyway, characterising such a population was out of the scope of our study which focuses only on the first group ('ab initio' monotherapies). Other limitations are intrinsic in the AHD-based design of the study, in particular lack of control of data collected for non-clinical purposes and misclassification biases; furthermore, clinical outcomes are lacking (absence of disease activity and radiological outcome data; specific causes of bDMARD failure or monotherapy prescription, such as patients' or physicians' preferences $^{22}$; possible alternative therapeutic schemes, including spacing of the bDMARD scheduled administration; different dosages of csDMARDs cotherapy). ${ }^{50}$ AHD reflects drug dispensing instead that the exact specialists' 'prescription' habit or the real patients' adherence, thus resulting in a difference between the rate of prescribed monotherapies and the rate of drug acquisition and use. Anyway, AHD are commonly considered a good instrument to estimate drug prescription and exposure ${ }^{51}$ and our data are in line with results from registries regarding monotherapy use in RA.

However, the RECORD study has some relevant strengths: its large sample size, allowing the examination of the effect of concomitant bDMARDs and csDMARDs, and the completeness of data without loss at follow-up. This is, to our knowledge, the first AHD-based study investigating different persistence rates in first-line biological monotherapies combining all bDMARDs approved as 'first-line' treatment.

In conclusion, our study supports the currently recommended use of bDMARDs in combination with csDMARDs, underlining a higher risk of drug withdrawal for TNFi monotherapy compared with combination and suggesting that, among bDMARDs, ETA should be preferred over INF-and to lesser extent ADA-when a first-line monotherapy is necessary. Despite univocal conclusions are not possible for non-TNFis, our results strengthen the positive influence of MTX, LFN or combination of csDMARDs in improving bDMARDs persistence.

Contributors Substantial contributions to study conception and design: $E S, A B$, $\mathrm{GC}, \mathrm{AZ}, \mathrm{MG}, \mathrm{CAS}$. Substantial contributions to acquisition of data: GC, $E S, A Z$, CAS. Substantial contributions to analysis and interpretation of data: $E S, A B, G C$, CAS. Drafting the article or revising it critically for important intellectual content: $E S, A B, G C, A Z, M G, C A S$. Final approval of the version of the article to be published: $E S, A B, G C, A Z, M G, C A S$. Agreement to be accountable for all aspects of the work in ensuring that questions related to the accuracy or integrity of any part of the work are appropriately investigated and resolved: $E S, A B, G C, A Z, M G$, CAS.

Funding This study was supported by the Italian Society for Rheumatology (SIR) as part of the Epidemiology Unit development programme.

Competing interests AB: Sanofi, Alfa-Wasserman and MG: Pfizer, Abbvie, MSD, Roche, BMS, Sanofi, Lilly, Novartis, Celgene received fees for sponsored lectures and/or participation in advisory boards.

Patient consent Not required.

Ethics approval The protocol was approved by the ethical committee of the Pavia University Hospital.
Provenance and peer review Not commissioned; externally peer reviewed.

Data sharing statement № additional information are available.

Open access This is an open access article distributed in accordance with the Creative Commons Attribution Non Commercial (CC BY-NC 4.0) license, which permits others to distribute, remix, adapt, build upon this work non-commercially, and license their derivative works on different terms, provided the original work is properly cited, appropriate credit is given, any changes made indicated, and the use is non-commercial. See: http://creativecommons.org/licenses/by-nc/4.0/.

\section{REFERENCES}

1. Smolen JS, Landewé R, Bijlsma J, et al. EULAR recommendations for the management of rheumatoid arthritis with synthetic and biological disease-modifying antirheumatic drugs: 2016 update. Ann Rheum Dis 2017;76:960-77.

2. Souto A, Maneiro JR, Gómez-Reino JJ. Rate of discontinuation and drug survival of biologic therapies in rheumatoid arthritis: a systematic review and meta-analysis of drug registries and health care databases. Rheumatology 2016;55:523-34.

3. O'Mahony A, Berg EL, John MR, et al. THU0526 Tocilizumab is less dependent than adalimumab on supplementary effects of methotrexate for immunoregulation: a biomap(r) profiling study. Ann Rheum Dis 2014;73:365.

4. Burmester GR, Kivitz AJ, Kupper $\mathrm{H}$, et al. Efficacy and safety of ascending methotrexate dose in combination with adalimumab: the randomised CONCERTO trial. Ann Rheum Dis 2015;74:1037-44.

5. Jani M, Barton A, Warren RB, et al. The role of DMARDs in reducing the immunogenicity of TNF inhibitors in chronic inflammatory diseases. Rheumatology 2014;53:213-22.

6. Garcês S, Demengeot J, Benito-Garcia E. The immunogenicity of anti-TNF therapy in immune-mediated inflammatory diseases: a systematic review of the literature with a meta-analysis. Ann Rheum Dis 2013;72:1947-55.

7. Choy E, Aletaha D, Behrens F, et al. Monotherapy with biologic disease-modifying anti-rheumatic drugs in rheumatoid arthritis: Table 1. Rheumatology 2016;70:kew271.

8. Burmester GR, Choy E, Kivitz A, et al. Low immunogenicity of tocilizumab in patients with rheumatoid arthritis. Ann Rheum Dis 2017;76:1078-85.

9. Dougados M, Kissel K, Conaghan PG, et al. Clinical, radiographic and immunogenic effects after 1 year of tocilizumab-based treatment strategies in rheumatoid arthritis: the ACT-RAY study. Ann Rheum Dis 2014;73:803-9.

10. Nash P, Nayiager S, Genovese MC, et al. Immunogenicity, safety, and efficacy of abatacept administered subcutaneously with or without background methotrexate in patients with rheumatoid arthritis: results from a phase III, international, multicenter, parallel-arm, openlabel study. Arthritis Care Res 2013;65:718-28.

11. van der Heijde D, Breedveld FC, Kavanaugh A, et al. Disease activity, physical function, and radiographic progression after longterm therapy with adalimumab plus methotrexate: 5-year results of PREMIER. J Rheumatol 2010;37:2237-46.

12. Mehta N, Schneider LK, McCardell E. Rheumatoid arthritis: selecting monotherapy versus combination therapy. J Clin Rheumatol 2017 (Published Online First: 18 Jan 2017).

13. Emery P, Sebba A, Huizinga TW. Biologic and oral disease-modifying antirheumatic drug monotherapy in rheumatoid arthritis. Ann Rheum Dis 2013;72:1897-904.

14. Listing J, Strangfeld A, Rau R, et al. Clinical and functional remission: even though biologics are superior to conventional DMARDs overall success rates remain low-results from RABBIT, the German biologics register. Arthritis Res Ther 2006;8:R66.

15. Lee SJ, Chang $\mathrm{H}$, Yazici $\mathrm{Y}$, et al. Utilization trends of tumor necrosis factor inhibitors among patients with rheumatoid arthritis in a United States observational cohort study. J Rheumatol 2009;36:1611-7.

16. Soliman MM, Ashcroft DM, Watson KD, et al. Impact of concomitant use of DMARDs on the persistence with anti-TNF therapies in patients with rheumatoid arthritis: results from the British Society for Rheumatology Biologics Register. Ann Rheum Dis 2011;70:583-9.

17. Carrara G, Scirè CA, Zambon A, et al. A validation study of a new classification algorithm to identify rheumatoid arthritis using administrative health databases: case-control and cohort diagnostic accuracy studies. Results from the RECord linkage On Rheumatic diseases study of the italian society for rheumatology. BMJ Open 2015;5:e006029.

18. Generali E, Carrara G, Selmi C, et al. Comparison of the risks of hospitalisation for cardiovascular events in patients with rheumatoid 
arthritis treated with tocilizumab and etanercept. Clin Exp Rheumatol. . 2018;Mar-Apr;36(2):310-313

19. Caprioli M, Carrara G, Sakellariou G, et al. Influence of aromatase inhibitors therapy on the occurrence of rheumatoid arthritis in women with breast cancer: results from a large population-based study of the Italian Society for Rheumatology. RMD Open 2017;3:e000523.

20. Barber C, Lacaille D, Fortin PR. Systematic review of validation studies of the use of administrative data to identify serious infections. Arthritis Care Res 2013;65:1343-57.

21. Deyo RA, Cherkin DC, Ciol MA. Adapting a clinical comorbidity index for use with ICD-9-CM administrative databases. J Clin Epidemiol 1992;45:613-9.

22. Pappas DA, Reed GW, Saunders K, et al. Characteristics associated with biologic monotherapy use in biologic-naive patients with rheumatoid arthritis in a us registry population. Rheumatol Ther 2015;2:85-96.

23. Jørgensen TS, Kristensen LE, Christensen R, et al. Effectiveness and drug adherence of biologic monotherapy in routine care of patients with rheumatoid arthritis: A cohort study of patients registered in the danish biologics registry. Rheumatology 2015;54:2156-65.

24. Heiberg MS, Koldingsnes W, Mikkelsen K, et al. The comparative one-year performance of anti-tumor necrosis factor $\alpha$ drugs in patients with rheumatoid arthritis, psoriatic arthritis, and ankylosing spondylitis: Results from a longitudinal, observational, multicenter study. Arthritis \& Rheumatism 2008;59:234-40.

25. Gabay C, Riek M, Scherer A, et al. Effectiveness of biologic DMARDs in monotherapy versus in combination with synthetic DMARDs in rheumatoid arthritis: data from the Swiss Clinical Quality Management Registry. Rheumatology 2015;54:1664-72.

26. Zhang J, Xie F, Delzell E, et al. Impact of biologic agents with and without concomitant methotrexate and at reduced doses in older rheumatoid arthritis patients. Arthritis Care Res 2015;67:624-32.

27. Scirè $C A$, Caporali R, Sarzi-Puttini $P$, et al. Drug survival of the first course of anti-TNF agents in patients with rheumatoid arthritis and seronegative spondyloarthritis: analysis from the MonitorNet database. Clin Exp Rheumatol 2013;31:857-63.

28. Orme ME, Macgilchrist KS, Mitchell S, et al. Systematic review and network meta-analysis of combination and monotherapy treatments in disease-modifying antirheumatic drug-experienced patients with rheumatoid arthritis: analysis of American College of Rheumatology criteria scores 20, 50, and 70. Biologics 2012;6:429-64.

29. Kristensen LE, Saxne T, Nilsson JA, et al. Impact of concomitant DMARD therapy on adherence to treatment with etanercept and infliximab in rheumatoid arthritis. Results from a six-year observational study in southern Sweden. Arthritis Res Ther 2006;8:R174.

30. Favalli EG, Pregnolato F, Biggioggero $\mathrm{M}$, et al. Twelve-year retention rate of first-line tumor necrosis factor inhibitors in rheumatoid arthritis: real-life data from a local registry. Arthritis Care Res 2016:68:432-9.

31. Frazier-Mironer A, Dougados M, Mariette X, et al. Retention rates of adalimumab, etanercept and infliximab as first and second-line biotherapy in patients with rheumatoid arthritis in daily practice. Joint Bone Spine 2014;81:352-9.

32. Iannone F, Gremese E, Atzeni F, et al. Longterm retention of tumor necrosis factor- $\alpha$ inhibitor therapy in a large italian cohort of patients with rheumatoid arthritis from the GISEA registry: an appraisal of predictors. J Rheumatol 2012;39:1179-84.

33. Jørgensen TS, Turesson C, Kapetanovic M, et al. EQ-5D utility, response and drug survival in rheumatoid arthritis patients on biologic monotherapy: A prospective observational study of patients registered in the south Swedish SSATG registry. PLoS One 2017;12:e0169946.

34. Weaver AL, Lautzenheiser RL, Schiff MH, et al. Real-world effectiveness of select biologic and DMARD monotherapy and combination therapy in the treatment of rheumatoid arthritis: results from the RADIUS observational registry. Curr Med Res Opin 2006;22:185-98.

35. Choy EH, Bernasconi C, Aassi M, et al. Treatment of rheumatoid arthritis with anti-tumor necrosis factor or tocilizumab therapy as first biologic agent in a global comparative observational study. Arthritis Care Res 2017:69:1484-94.

36. Buckley F, Finckh A, Huizinga TW, et al. Comparative efficacy of novel dmards as monotherapy and in combination with methotrexate in rheumatoid arthritis patients with inadequate response to conventional dmards: a network meta-analysis. J Manag Care Spec Pharm 2015;21:409-23.

37. Batticciotto A, Ravasio R, Riva M, et al. Efficacy and treatment costs of monotherapy with bdmards in the treatment of rheumatoid arthritis in patients intolerant to or inappropriate to continue treatment with methotrexate. Adv Ther 2016;33:1360-73.

38. Huizinga TW, Conaghan PG, Martin-Mola E, et al. Clinical and radiographic outcomes at 2 years and the effect of tocilizumab discontinuation following sustained remission in the second and third year of the ACT-RAY study. Ann Rheum Dis 2015;74:35-43.

39. Mueller RB, Graninger W, Sidiropoulos P, et al. Median time to low disease activity is shorter in tocilizumab combination therapy with csDMARDs as compared to tocilizumab monotherapy in patients with active rheumatoid arthritis and inadequate responses to csDMARDs and/or TNF inhibitors: sub-analysis of the Swiss and Austrian patients from the ACT-SURE study. Clin Rheumatol 2017:36:2187-92.

40. Kaneko Y, Atsumi T, Tanaka Y, et al. Comparison of adding tocilizumab to methotrexate with switching to tocilizumab in patients with rheumatoid arthritis with inadequate response to methotrexate: 52-week results from a prospective, randomised, controlled study (SURPRISE study). Ann Rheum Dis 2016;75:1917-23.

41. Weinblatt ME, Kremer J, Cush J, et al. Tocilizumab as monotherapy or in combination with nonbiologic disease-modifying antirheumatic drugs: Twenty-four-week results of an open-label, clinical practice study. Arthritis Care Res 2013;65:362-71.

42. Alfonso-Cristancho R, Armstrong N, Arjunji R, et al. Comparative effectiveness of biologics for the management of rheumatoid arthritis: systematic review and network meta-analysis. Clin Rheumatol 2017;36:25-34.

43. Gabay C, Emery P, van Vollenhoven R, et al. Tocilizumab monotherapy versus adalimumab monotherapy for treatment of rheumatoid arthritis (ADACTA): a randomised, double-blind, controlled phase 4 trial. Lancet 2013;381:1541-50.

44. Manders SH, Kievit W, Jansen TL, et al. Effectiveness of tumor necrosis factor inhibitors in combination with various csdmard in the treatment of rheumatoid arthritis: data from the DREAM Registry. $J$ Rheumatol 2016;43:1787-94.

45. De Stefano R, Frati E, Nargi F, et al. Comparison of combination therapies in the treatment of rheumatoid arthritis: leflunomide-antiTNF-alpha versus methotrexate-anti-TNF-alpha. Clin Rheumatol 2010;29:517-24.

46. Flendrie M, Creemers MC, Welsing PM, et al. The influence of previous and concomitant leflunomide on the efficacy and safety of infliximab therapy in patients with rheumatoid arthritis; a longitudinal observational study. Rheumatology 2005;44:472-8.

47. Wendler J, Burmester GR, Sörensen $\mathrm{H}$, et al. Rituximab in patients with rheumatoid arthritis in routine practice (GERINIS): six-year results from a prospective, multicentre, non-interventional study in 2,484 patients. Arthritis Res Ther 2014;16:R80.

48. Chatzidionysiou K, Lie E, Nasonov E, et al. Effectiveness of diseasemodifying antirheumatic drug co-therapy with methotrexate and leflunomide in rituximab-treated rheumatoid arthritis patients: results of a 1-year follow-up study from the CERERRA collaboration. Ann Rheum Dis 2012;71:374-7.

49. Acurcio FA, Machado MAA, Moura CS, et al. Medication persistence of disease-modifying antirheumatic drugs and anti-tumor necrosis factor agents in a cohort of patients with rheumatoid arthritis in Brazil. Arthritis Care Res 2016;68:1489-96.

50. Krishnan E, Fries JF. Measuring effectiveness of drugs in observational databanks: promises and perils. Arthritis Res Ther 2004;6:41-4.

51. Cadarette SM, Wong L. An introduction to health care administrative data. Can J Hosp Pharm 2015;68:232-7. 